\title{
Metastatic Cardiac Tumor from Precursor B Cell Lymphoblastic Lymphoma with a Mediastinal Mass: A Case Report of a 12-Years-Old African Girl
}

\author{
Dorah Nampijja ${ }^{1,2 *}$, Aliku Twalib ${ }^{1}$, Kumbakumba Elias ${ }^{2}$ and Sulaiman Lubega ${ }^{1}$ \\ ${ }^{1}$ Uganda Heart Institute, Mulago Complex, Kampala, Uganda \\ ${ }^{2}$ Mbarara University of Science and Technology, Mbarara, Uganda
}

*Corresponding author: Dorah Nampijja, Uganda Heart Institute, Mulago Complex, Kampala, Mbarara University of Science and Technology, Mbarara, Uganda

\begin{abstract}
Introduction: Cardiac involvement in metastatic tumors is not widely investigated. Commonest site of cardiac involvement is the pericardium. However, diffuse metastasis to the myocardium and endocardium is uncommon. Striated cardiac muscle, fast blood flow within the heart and unique lymphatic drainage make malignant metastasis to the heart rare. Cardiac metastasis is a late manifestation of malignancy and most of the cases are diagnosed on Post-mortem. We describe a case of metastatic Pre B-Lymphoblastic Lymphoma in a child who presented with heart failure.
\end{abstract}

Case presentation: A 12-years-old girl was presented with an acute history of fever, cough and chest pain. She had associated difficulty in breathing, lower limb swelling and exercise intolerance. Examination findings were of a very ill child, afebrile, mild pallor, no jaundice, no lymphadenopathy. Had tachypnea, severe subcostal recession and bilateral reduced air entry, a tender hepatomegaly and grade III pedal edema, diminished peripheral pulses, excessive sweating, altered mental state and central cyanosis. Heart sounds were of normal intensity and had no murmurs.

CT scan showed a large solid irregular infiltrative mediastinal mass encasing the superior vena cava, ascending aorta, trachea, descending aorta, right and left main bronchus and the cardiac chambers.

Transthoracic echocardiograph showed normal sized heart chambers with grossly thickened myocardial tissue. She had multiple infiltrative masses in the lateral walls of the right ventricle and left ventricle. She had a large fixed mass in the right atrium. Both parietal and visceral pericardia were thickened with thick pericardial effusion. Cytology revealed Precursor B cell Lymphoblastic lymphoma.
Child was initiated on chemotherapy excluding anthracycline due to the poor systolic function. She got a recurrence and died eight months after initiation of chemotherapy.

Conclusions: We report a case of a metastatic cardiac tumor from a Pre B- cell lymphoblastic lymphoma presenting with heart failure.

\section{Introduction}

Precursor B lymphoblastic Lymphoma (B-LBL) is a rare type of lymphoblastic lymphoma a highly aggressive subtype of Non Hogkin's lymphoma at less than $10 \%$ [1]. They are lymphoid neoplasm of immature precursor cells of the B cell lineage that express B-cell markers [2]. Morphologically, indistinguishable from acute lymphoblastic leukemia, LBL is characterized by a lymphoid mass and limited or no bone marrow and peripheral blood involvement. Precussor B-Lymphoblastic lymphomas are mostly localized to peripheral lymphnodes, extranodal sites like skin, soft tissue and bone [3].

Cardiac involvement due to lymphomas is not widely investigated. Symptoms are usually non specific to the primary tumor, difficult to diagnose almost always associated with advanced disease and are fatal $[4,5]$. A lot of emphasis has been put on cardiomyopathy related to chemotherapy. Commonest site of cardiac involvement is the pericardium. Cardiac metastasis is a late manifestation of malignancy and most of the cases are diagnosed on post-mortem [6].

Citation: Nampijja D, Twalib A, Elias K, Lubega S (2019) Metastatic Cardiac Tumor from Precursor B Cell Lymphoblastic Lymphoma with a Mediastinal Mass: A Case Report of a 12-Years-Old African Girl. Int Arch Cardiovasc Dis 3:017

Accepted: April 13, 2019; Published: April 15, 2019

Copyright: (c) 2019 Nampijja D, et al. This is an open-access article distributed under the terms of the Creative Commons Attribution License, which permits unrestricted use, distribution, and reproduction in any medium, provided the original author and source are credited. 
We describe a case of metastatic precursor B-Lymphoblastic Lymphoma with a mediastinal mass in a child who presented with heart failure, cyanosis and shock.

\section{Case Report}

A 12-years-old girl who presented with acute history (2 weeks) lower limb swelling, difficulty in breathing, cough and chest pain with associated exercise intolerance. Past Medical history was not significant. She had no immune suppression or history of weight loss or fevers. Examination findings were of a very ill child, axillary temperature $36.7{ }^{\circ} \mathrm{C}$, mild pallor, no jaundice, Grade III pedal oedema. She had no peripheral lymphadenopathy. She was very tachypnoec with a respiratory rate of $56 \mathrm{bpm}$, severe subcostal recession and bilateral reduced air entry. She had a tender hepatomegaly $8 \mathrm{~cm}$ below the costal margin. Had no murmurs, heart sound were of normal intensity. The child would get episodes circulatory collapse worsened when lying flat characterized by diminished peripheral pulses, excessive sweating, altered mental state and central cyanosis.

While in hospital, the child underwent a series of diagnostic investigations. In view of the pleural effusion she was extensively investigated for tuberculosis with sputum analysis (microscopy and gene expert, pleural centesis with analysis, tuberculin skin test (mantoux), serial full blood counts, bone marrow aspiration and analysis which were normal.

She was initially treated for severe pneumonia with broad spectrum antibiotics (Ceftriaxone and Gentamycin) with no improvement. She was later empirically treated for tuberculosis, however, her clinical condition continued to deteriorate.
In view of difficulty in breathing and edema, an echocardiogram was ordered.

Transthoracic echocardiograph showed normal sized heart chambers with grossly thickened myocardial tissue. She had fractional shortening of $13 \%$ (Figure 1) with poor RV contractility, a TAPSE of $11 \mathrm{~mm}$.

She had multiple infiltrative masses in the lateral walls of the right ventricle and left ventricle with poor contractility of the ventricles. She had a large fixed mass in the right atrium. Both parietal and visceral pericardia were thickened with thick pericardial effusion. There was involvement of the lateral wall of the left ventricle with sentinel lesion at the hinge of the mitral valve (Figure 2 and Figure 3).

CT scan showed a large solid irregular infiltrative mediastinal mass encasing the superior venacava, ascending aorta, trachea, descending aorta, right and left main bronchus and the cardiac chambers (Figure 4).

Diagnosis of pre B LBL was made using flow cytometry on the pleural fluid.

Child was initiated on induction chemotherapy (three drugs instead of four; Dexamethasone, Vincristine, Asparaginase). She also received intrathecal chemotherapy for CNS prophylaxis. The Anthracycline was excluded due to low ejection fraction (13\%). She also received Frusemide and Digoxin in view of heart failure and systolic dysfunction.

By day 28 of induction, her symptoms of heart failure and the cardiac masses had resolved. However, child got a recurrence with similar presentation of difficulty in breathing and convulsions. Palliative care was initiated and she died at home 8 months after the initial diag-

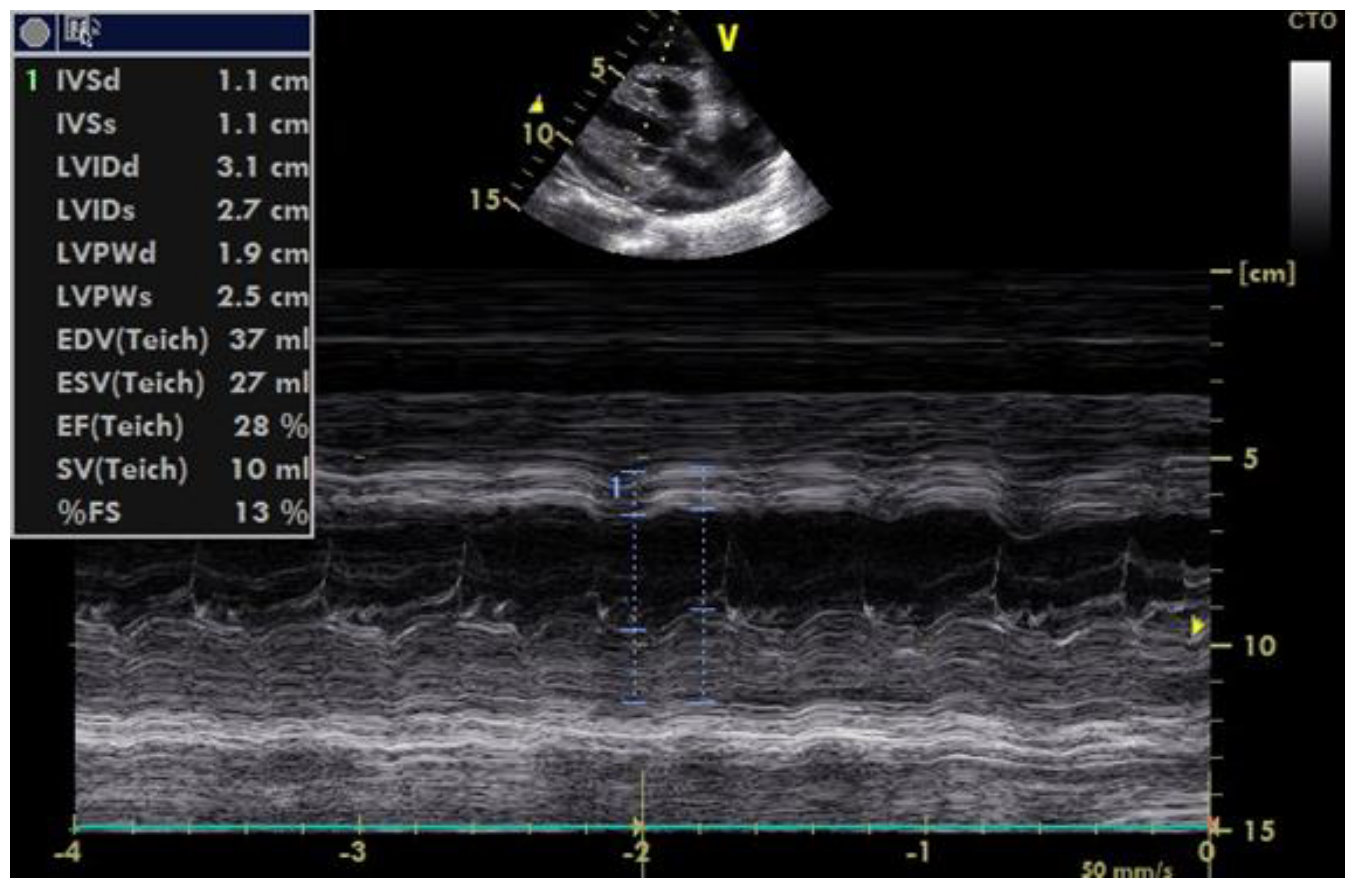

Figure 1: An image showing a low ejection fraction, thickened infiltrated ventricular walls and a pericardial effusion. 


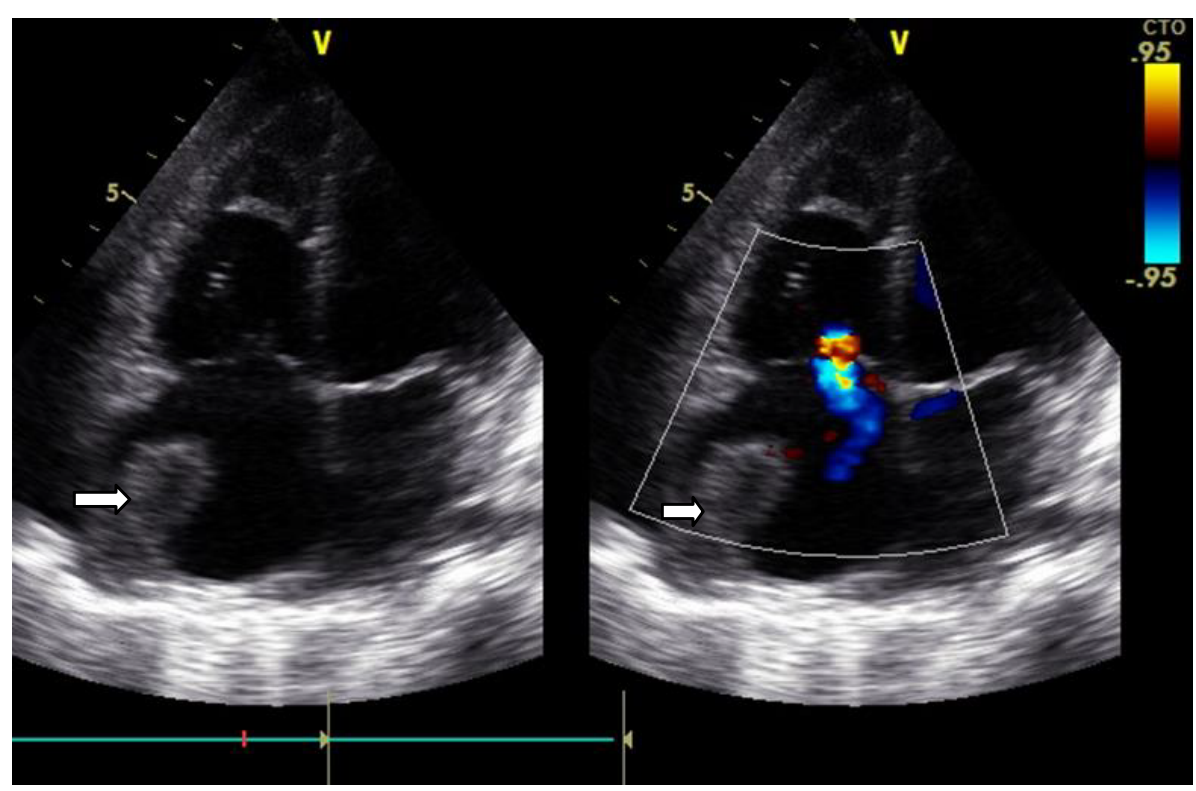

Figure 2: An image showing the tumor at the roof of the right atrium (arrows) and mild regurgitation of tricuspid valve.

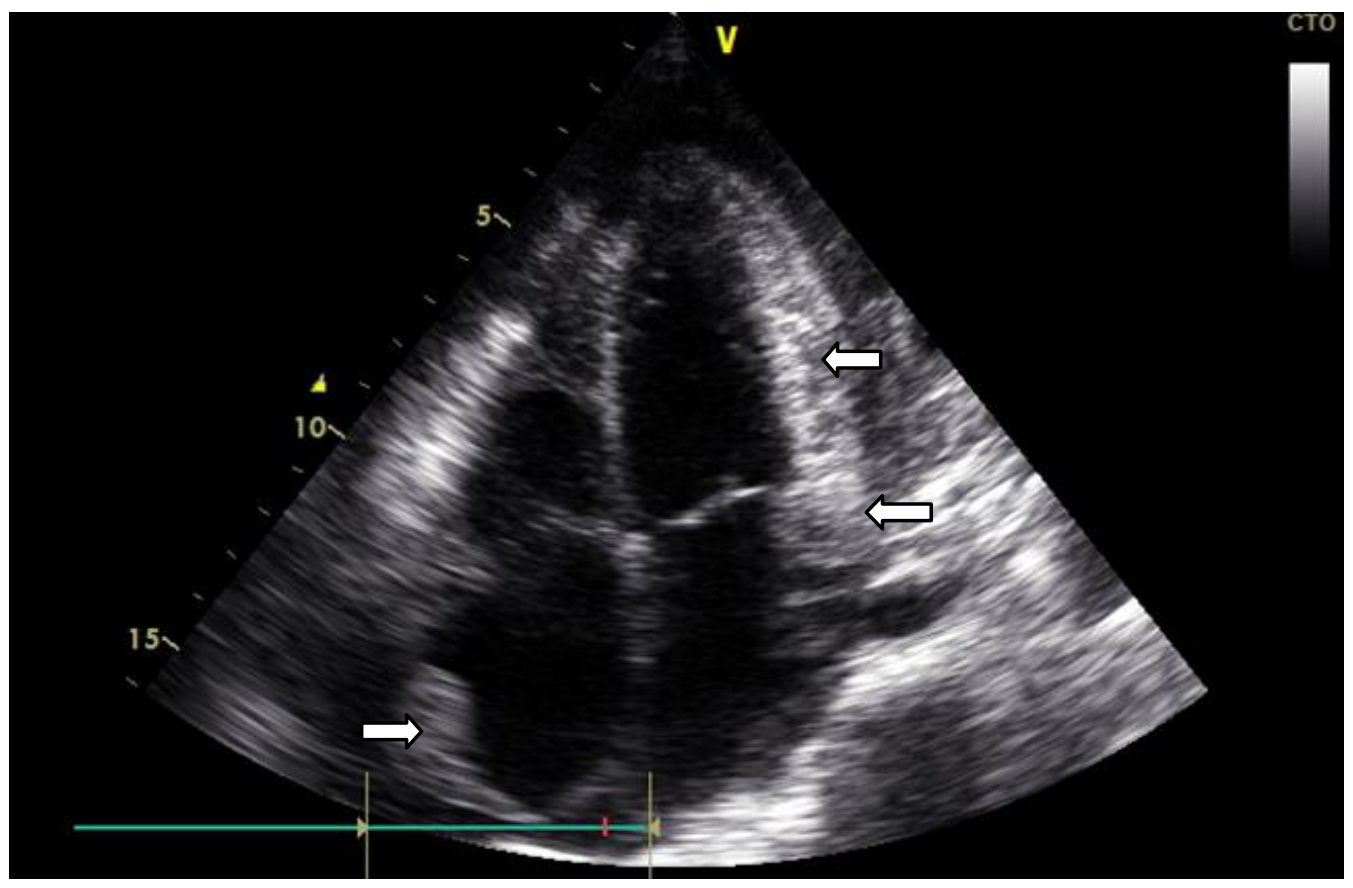

Figure 3: An image showing the tumor infiltration of the right atrial wall and lateral wall of the left ventricle.
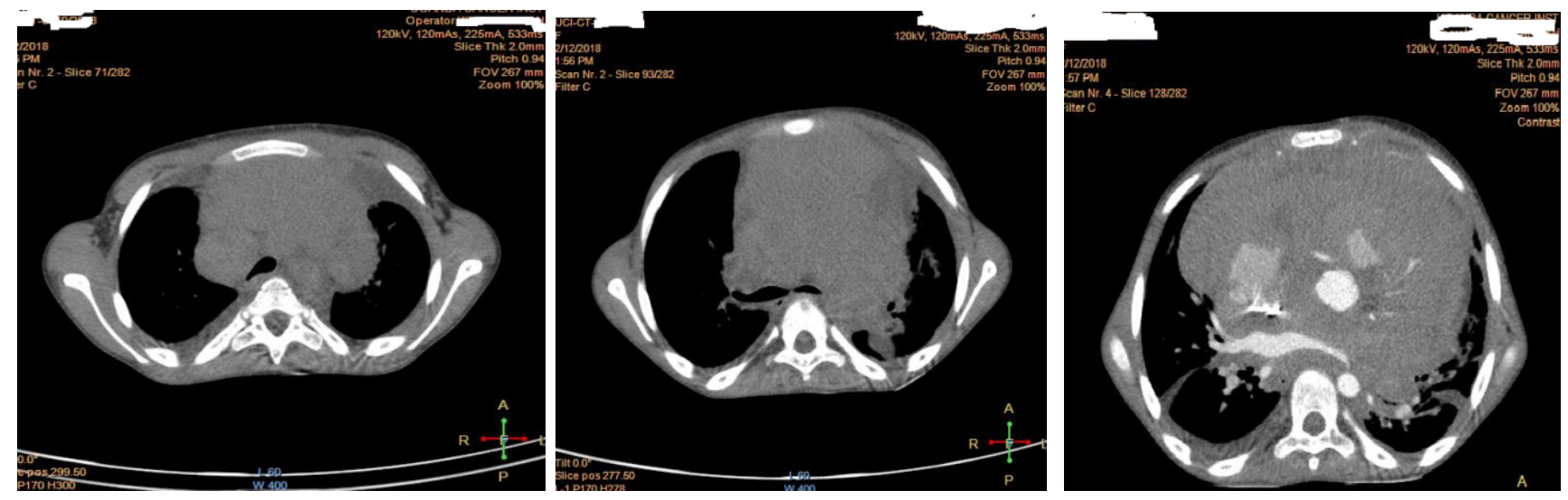

Figure 4: CT scan showing a large mediastinal mass involving the aorta, superior venacava and cardiac chambers. 
nosis and initiation of chemotherapy. Post-mortem was not done.

\section{Discussion}

Most cardiac tumors are benign in nature [7]. Primary malignant cardiac tumors are much more rare in children $[8,9]$ the majority being due to metastatic tumors. Diagnosis of cardiac tumors is challenging since symptoms may be masked by the underlying tumor [10-12]. Heart failure is an uncommon presentation of lymphoma in children.

Lymphomas are malignant blood cancers and third most common cancer in children. They exist in two categories; Hodgkin and non-Hodgkin lymphomas. They may seem similar diseases, but they are however different disease entities with specific clinical manifestations and different treatment.

Precursor B-LBL is largely a disease of children and young adults, characterized by rapid growth of a mass involving lymph nodes, skin, bone, head, neck and retroperitoneal space. B-LBL is rarely associated with mediastinal masses. The case we presented did not have bone marrow or lymph node involvement. She had a large mediastinal mass that extended to the cardiac chambers and great vessels. The tumor infiltration into the myocardium and the great blood vessels resulted into a cardiomyopathy with poor contractility of the heart and low ejection fraction which may explain the heart failure. Possible obstruction of the tumor in the right atrium could explain the circulatory collapse.

Cardiac involvement, whether primary or secondary, is usually associated with poor prognosis [13]. It has variant presentation ranging from mild pericardial effusion to cardiogenic shock. Most diagnoses are made on postmortem. The non specific presentation made the diagnosis of the tumor difficult and delayed which is similar to what is encountered in other cases of cardiac infiltration by malignant lymphomas [5].

Cardiac tumors present a challenge in the management especially when presenting with cardiomyopathy. The choice of chemotherapy becomes very difficult given that most chemotherapy is cardiotoxic and associated with cardiomyopathy.

This dilemma gets compounded in developing countries where access to other alternative treatment modalities is limited.

\section{Conclusion}

We present an uncommon presentation of pre B lymphoblastic lymphoma highlighting one of the various ways lymphomas present in children.

\section{Declaration}

Authors declare no conflict of interest.

Written informed consent for publication of their clinical details and clinical images was obtained from the parents of the patient. A copy of the consent form is available for review by the editor of this journal.

\section{Acknowledgement}

We would like to acknowledge the patient and her family.

\section{References}

1. Geethakumari PR, Hoffmann MS, Pemmaraju N, Hu S, Jorgensen JL, et al. (2014) Extramedullary B lymphoblastic leukemia/lymphoma (B-ALL/B-LBL): A diagnostic challenge. Clin Lymphoma Myeloma Leuk 14: e115-e118.

2. Campo E, Swerdlow SH, Harris NL, Pileri S, Stein $\mathrm{H}$, et al. (2011) The 2008 WHO classification of lymphoid neoplasms and beyond: Evolving concepts and practical applications. Blood 117: 5019-5032.

3. Lin P, Jones D, Dorfman DM, Medeiros LJ (2000) Precursor B-cell lymphoblastic lymphoma: A predominantly extranodal tumor with low propensity for leukemic involvement. Am J Surg Pathol 24: 1480-1490.

4. Moustafa S, Patton DJ, Alvarez N, Prieur T, Connelly MS, et al. (2014) Unusual cardiac infiltration in diffuse large B-cell lymphoma. J Cardiovasc Ultrasound 22: 160-161.

5. Vinicki JP, Cianciulli TF, Farace GA, Saccheri MC, Lax JA, et al. (2013) Complete regression of myocardial involvement associated with lymphoma following chemotherapy. World J Cardiol 5: 364-368.

6. Chinen K, Izumo T (2005) Cardiac involvement by malignant lymphoma: A clinicopathologic study of 25 autopsy cases based on the WHO classification. Ann Hematol 84: 498505.

7. Ying L, Lin R, Gao Z, Qi J, Zhang Z, et al. (2016) Primary cardiac tumors in children: A center's experience. J Cardiothorac Surg 11: 52.

8. Beghetti M, Gow RM, Haney I, Mawson J, Williams WG, et al. (1997) Pediatric primary benign cardiac tumors: A 15year review. Am Heart J 134: 1107-1114.

9. Mariano A, Pita A, León R, Rossi R, Gouveia R, et al. (2009) Primary cardiac tumors in children: A 16-year experience. Rev Port Cardiol 28: 279-288.

10. Nadas AS, Ellison RC (1968) Cardiac tumors in infancy. Am J Cardiol 21: 363-366.

11. Fernandes F, Soufen HN, lanni BM, Arteaga E, Ramires FJ, et al. (2001) Primary neoplasms of the heart. Clinical and histological presentation of 50 cases. Arq Bras Cardiol 76: 231-237.

12. Reynen K, Strasser RH, Köckeritz U (2004) Metastases to the heart. Annals of Oncology 15: 375-381.

13. Tao TY, Yahyavi-Firouz-Abadi N, Singh GK, Bhalla $S$ (2014) Pediatric cardiac tumors: Clinical and imaging features. Radiographics 34: 1031-1046. 